\title{
Computational Cardiology: The Heart of the Matter
}

\author{
Natalia A. Trayanova \\ Department of Biomedical Engineering and Institute for Computational Medicine, Johns Hopkins University, \\ 3400 North Charles Street, Hackerman Hall Room 216, Baltimore, MD 21218, USA \\ Correspondence should be addressed to Natalia A. Trayanova, ntrayanova@jhu.edu
}

Received 16 August 2012; Accepted 6 September 2012

Academic Editors: T. Ohe and A. Szekely

Copyright ( $) 2012$ Natalia A. Trayanova. This is an open access article distributed under the Creative Commons Attribution License, which permits unrestricted use, distribution, and reproduction in any medium, provided the original work is properly cited.

\begin{abstract}
This paper reviews the newest developments in computational cardiology. It focuses on the contribution of cardiac modeling to the development of new therapies as well as the advancement of existing ones for cardiac arrhythmias and pump dysfunction. Reviewed are cardiac modeling efforts aimed at advancing and optimizing existent therapies for cardiac disease (defibrillation, ablation of ventricular tachycardia, and cardiac resynchronization therapy) and at suggesting novel treatments, including novel molecular targets, as well as efforts to use cardiac models in stratification of patients likely to benefit from a given therapy, and the use of models in diagnostic procedures.
\end{abstract}

\section{Introduction}

The iterative interaction between experimentation and simulation has long played a central role in the advancement of biological sciences. Among computational models of the various physiological systems, the heart is the most highly advanced example of a virtual organ, capable of integrating data at multiple scales, from genes to the whole organ [1]. State-of-the-art whole-heart models of electrophysiology and electromechanics are currently being used to study a wide range of mechanisms in the workings of the normal and the diseased heart $[2,3]$. The focus of this paper is on the contribution of heart computational models to the treatment of the diseased heart, that is, on the computational medicine aspect of cardiac modeling applications. Reviewed below are cardiac modeling efforts aimed at advancing and optimizing existent therapies for cardiac disease and at suggesting novel treatments, including novel molecular targets, as well as efforts to use cardiac models in stratification of patients likely to benefit from a given therapy, and the use of models in diagnostic procedures

\section{Modeling Ventricular Arrhythmias: From Mechanisms to the Clinic}

Modeling arrhythmias in the whole heart to reveal mechanisms and suggest better treatments has become one of the most important hallmarks in the utilization of biophysically detailed computational modeling of the heart. A number of ventricular models have focused on arrhythmia dynamics, and specifically on the self-sustained reentrant propagation of complex 3D waves in the ventricles. Historically, these were the first applications of ventricular modeling. Ventricular modeling studies have revealed important aspects of reentrant arrhythmias, among which the dynamic characteristics of ventricular fibrillation (VF), and the role of alternans and restitution in arrhythmogenesis.

Ventricular models have been used extensively in characterizing VF dynamics $[4,5]$. Particularly interesting are the studies on human hearts [5], which revealed that human VF is driven by a small number of reentrant sources and is thus much more organized than VF in animal hearts of comparable size; the human action potential duration (APD) was found responsible for the specific VF dynamics in the human heart. Furthermore, a number of simulation studies have focused on alternans and their role in arrhythmogenesis. Electrical alternans, which are beat-to-beat changes in APD, have long been recognized as a precursor to the development of VF. Alternans can be concordant with the entire tissue experiencing the same phase of oscillation, or discordant, with opposite-phase regions distributed throughout the tissue. Over the last decade, much emphasis has been placed on the restitution curve slope as a major factor in both the onset 
of arrhythmias following the development of discordant alternans, and the dynamic destabilization of reentrant waves leading to the transition of ventricular tachycardia (VT) into VF. In what has become known as the restitution hypothesis, flattening the APD restitution curve is postulated to inhibit alternans development and subsequent conduction block, and prevent the onset of VF [6]. Simulation studies employing ventricular models [7-11] have made important contributions to ascertaining the intricate set of mechanisms by and the conditions under which steep APD restitution could lead to VF onset. These include the dynamical transition from concordant to discordant alternans [8], the role of electrotonic and memory effects in suppressing alternans and stabilizing reentrant waves, and the effect of heterogeneous restitution properties on human VF $[10,11]$. A recent ventricular modeling study by McDowell et al. [12] employed a novel 3D computational model of the chronically infarcted rabbit ventricles to characterize the arrhythmogenic substrate resulting from myofibroblast infiltration. Furthermore, a number of ventricular simulation studies have incorporated the Purkinje system [13], focusing on the role of the Purkinje system in ventricular arrhythmias. The study by Deo et al. [14] determined the conditions for arrhythmogenesis due to early afterdepolarizations (EADs) originating in Purkinje cells; the latter are known to be more vulnerable to EADs than ventricular myocytes. Finally, simulation of ventricular arrhythmia mechanisms in the setting of regional ischemia [15-17] has characterized the substrate for ischemia phase $1 \mathrm{~B}$ arrhythmias by examining how the interplay between different degrees of hyperkalemia in the surviving layers, and the level of cellular uncoupling between these layers and the midmyocardium combine with the specific geometry of the ischemic zone in the ventricles to result in reentrant arrhythmias (Figure 1(a), simulation of arrhythmia in a model of regional ischemia in the rabbit ventricles). Computer simulations of cardiac ischemia and the corresponding body surface potentials have also been used to determine how the extent of the ischemic zone is reflected in the 12-lead ECG.

Recent years have witnessed revolutionary advances in imaging, including ex vivo structural and diffusion tensor (DT) magnetic resonance imaging (MRI) that facilitate acquisition of the intact structure of explanted hearts with high resolution. Leveraging on these advances, a new generation of whole-heart image-based models with unprecedented detail have emerged $[18,19]$. Such ex vivo heart models are currently being used, in combination with experimental electrophysiological data, to provide better understanding of the role of the individual morphology of the infarct region in the generation and maintenance of infarct-related VT, the most frequent clinical ventricular arrhythmia, present in $64 \%$ of patients with ventricular rhythm disorder and in $89 \%$ of patients with sudden cardiac death [20]. A recent MRI-based ventricular modeling study by McDowell et al. [12] employed a 3D computational model of the chronically infarcted rabbit ventricles to characterize the arrhythmogenic substrate resulting from myofibroblast infiltration. It was found that myofibroblasts at low densities do not alter arrhythmia propensity, while at intermediate densities, myofibroblasts cause action potential shortening and exacerbate arrhythmia propensity. Interestingly, at high densities, myofibroblasts were found to protect against arrhythmia by causing resting depolarization and blocking propagation. Using a model of the infracted pig ventricles reconstructed from ex-vivo MRI and DTMRI data, the study by Pop et al. [21] demonstrated a good correspondence between in silico and experimental electroanatomical voltage maps and was able to successfully predict infarct-related VT inducibility after programmed electrical stimulation. Arevalo et al. $[22,23]$ examined the role of the extent of the infarct border zone in arrhythmogenesis (Figure 1(b), simulation of arrhythmia in MRI-based model of an infracted canine heart), establishing that a minimum volume of remodeled tissue is needed for VT maintenance and demonstrating that the organizing center of infarct-related VT is located within the infarct border zone, regardless of the pacing site from which VT is induced. Such simulation methodology could have a major clinical impact in predicting the optimal targets of catheter ablation of infarct-related VT in individual hearts, should the methodology be able to reconstruct patent hearts from clinical imaging data and evaluate the 3D patterns of infarct-related VT [24] in the patient. The first attempts in this direction have already been made. Figure 2 presents a simulation of arrhythmia in a patientspecific model of the infracted ventricles; it shows both models generation for the clinical MR scans of the patient heart, as well as the infarct-related ventricular tachycardia. The study by $\mathrm{Ng}$ et al. [25] demonstrated the feasibility of using simulations to predict VT circuits. Relan et al. [26] used a hybrid X-ray and MR environment to image a patient heart, which was further personalized with voltage measurements. The results demonstrated that the heart model could successfully be used to assess infract-related VT inducibility from sites not accessible in the clinic. Further translation of ventricular simulations in the clinic will be facilitated by the development of methodologies to estimate patient-specific fiber orientations from clinical MRI scans $[27,28]$ (Figure 2(d)).

\section{Modeling Atrial Arrhythmogenesis}

Computational modeling of the electrophysiology of the human atria is also becoming an important component in the evaluation and advancement of therapeutic strategies, as recent state-of-the-art biophysically detailed models can accurately simulate the complex spatio-temporal dynamics of atrial arrhythmias. Similar to ventricular modeling methodology, biophysically detailed atrial models are reconstructed from MRI [30] and CT [31] scans, as well as datasets resulting from the Visible Human project [32-34]. Obtaining accurate fiber orientation for the atria, however, has proved more challenging than that in the ventricles, as applying techniques such as diffusion tensor imaging to the atria's thin walls is difficult.

Atrial models have been used to determine the arrhythmogenicity of substrates due to ionic remodeling [35] or electrophysiological heterogeneity [36]. Modeling studies 


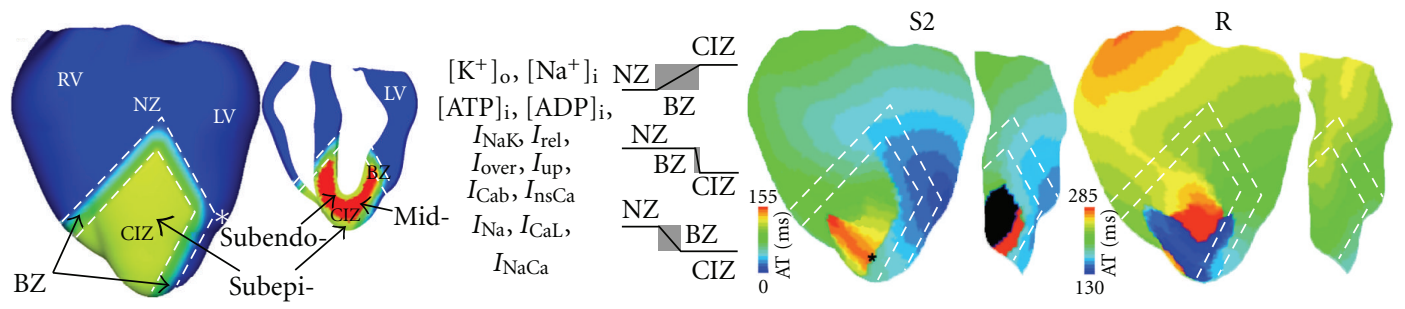

(a)

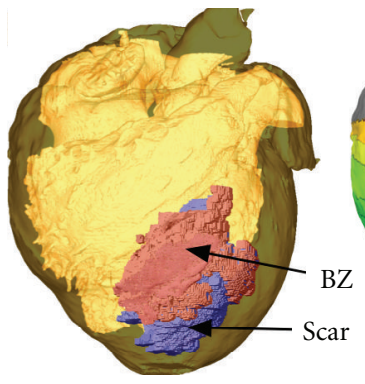

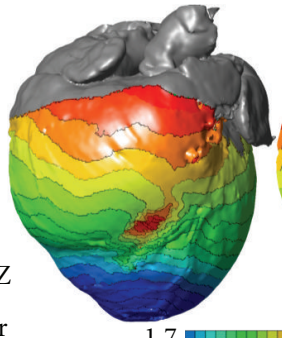

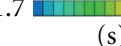

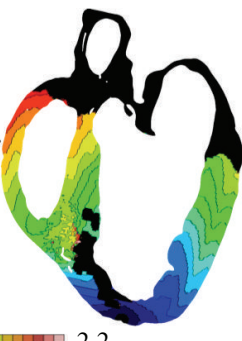

(s)

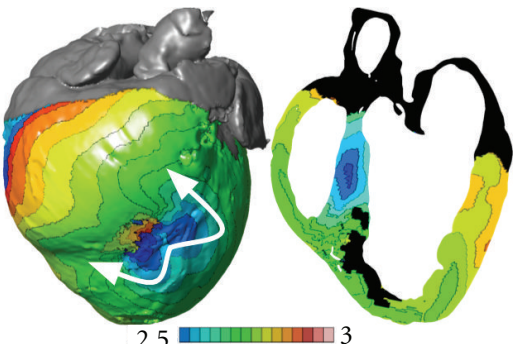

(s)

(b)

FIGURE 1: Modeling arrhythmias in ischemia and infarction. (a) Left: model of ischemia phase 1b following LAD occlusion in the rabbit heart, with central ischemic zone (CIZ) and border zone (BZ). Colors distinguish the regions. White dashes outline BZ. Asterisk indicates the stimulus site. Border zones for different ischemic parameters are also shown. Right: generation of reentry in the subepicardium following propagation of premature stimulus in myocardium uncoupled from the surviving endo- and epicardium. Activation maps on the anterior epicardial surface and in a cross-section across the LV are shown. S2 and R refer to activation maps for the premature and the first reentrant beats, respectively. Black asterisk indicates reentry exit site. Figure modified from [15]. (b) Infarct-related VT in the canine heart. Left panel: MRI-based model of the infracted canine heart with scar and BZ. Remaining panels: activation maps of VT following programmed stimulation. Arrows indicate direction of propagation. Figure reprinted from [2].

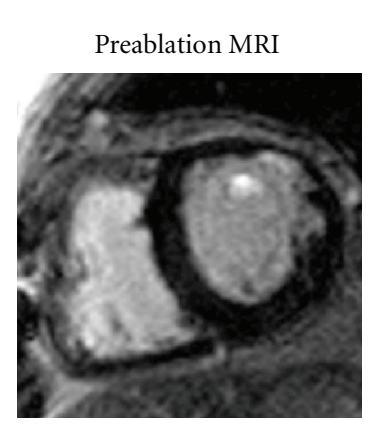

(a)

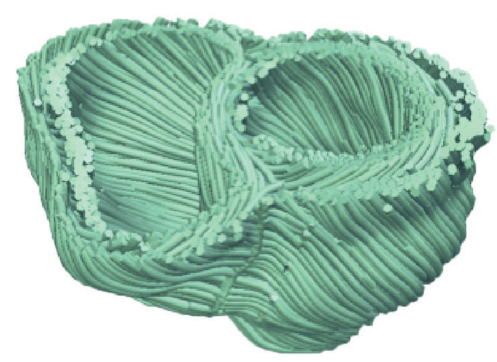

Fibers

(d)

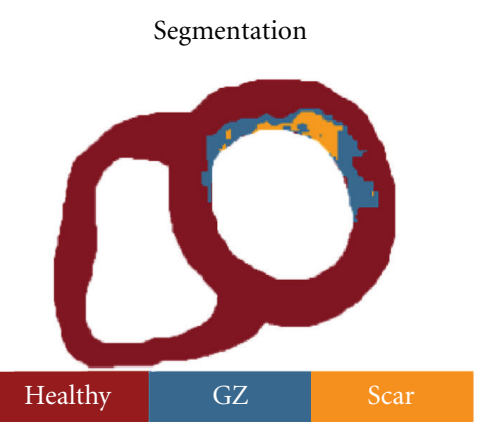

(b)

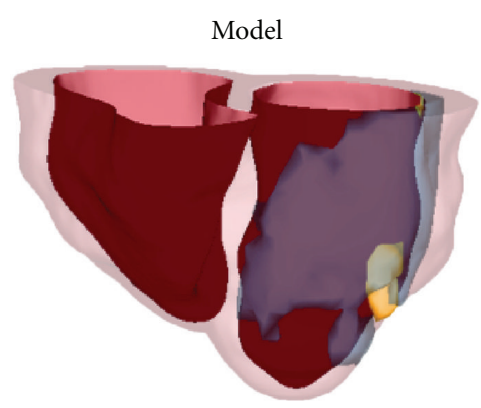

(c)

In silico VT

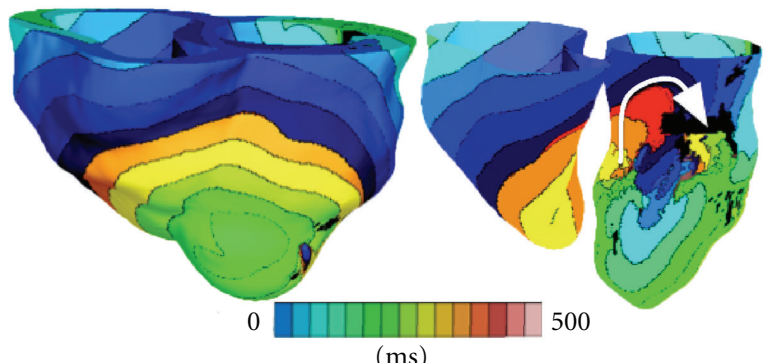

(e)

Figure 2: (a), (b) Clinical MRI scan of an infarcted patient heart and the corresponding segmentation. (c) 3D geometric model of the patient heart with the epicardium and the infarct border zone rendered semitransparent. (d) Estimated fiber orientations. (e) Simulated activation map of ventricular tachycardia (VT) revealing reentry on the left ventricular endocardium. VT frequency is $3.05 \mathrm{~Hz}$. Figure modified from [29]. 
have also ascertained the role of the autonomic nervous system in atrial arrhythmias, finding that heterogeneous densities of an acetylcholine-driven potassium current lead to a cholinergic form of AF [37]. Atrial modeling has explored the mechanism behind pulmonary vein ectopy, known to initiate AF, finding that activation may arise from differences in resting potential between myocytes and coupled fibroblasts [38]. Other lines of study, pursued with complex atrial models, include representation of intrinsic structure and APD heterogeneity [32, 39-42] and simulated ablation [43-45].

Contemporary atrial models allow for realistic simulation of ECG signals [46], monophasic action potentials [47], and electrograms [48], serving as an important tool for analyzing the etiologies that underlie electrical signals obtained clinically. For example, simulation studies of bipolar electrograms in atrial tissue have found that fibroblast proliferation and microscale obstacles (such as collagenous septa) may be responsible for the fractionation of electrograms [49] during atrial fibrillation (AF), signals which guide catheter ablation of AF. Studies involving simulation of ECG signals in atrial models have helped optimize procedures such as hemodialysis therapy (which can cause AF) [50] and predicting the optimal position and direction of one-channel bipolar ECGs [51]. Full utilization of multiscale atrial models in the clinic for preventative, diagnostic, or therapeutic purposes will require the generation of patient-specific atrial models; early efforts in this direction are already underway $[52,53]$. Figure 3 presents the first patient-specific model of $\mathrm{AF}$ in the fibrotic atria; panels include the atrial model and the reentrant arrhythmia induced as a result of pulmonary vein ectopy.

\section{Electromechanical Modeling of Heart Function and Its Application to Cardiac Resynchronization Therapy}

Biophysically detailed electromechanical models of the heart, which represent the most sophisticated models of the heart developed thus far [55], have also been harnessed in the development of therapies for pump dysfunction. Cardiac resynchronization therapy (CRT) employs biventricular pacing to recoordinate the contraction of the failing heart. Electromechanical modeling studies have provided insight into the mechanisms that govern CRT efficacy. Kerckhoffs and coworkers $[56,57]$ have demonstrated that improvement of ventricular function following CRT in the failing heart with left bundle branch block (LBBB) is diminished with increasing infarct size and that infarct location also affects the response to CRT. Niederer et al. [58] revealed that the hemodynamic benefit from CRT is improved when length-dependent tension regulation is attenuated; the study suggested that a compromised Frank-Starling mechanism (the organ-level equivalent of length-dependent tension regulation) could be a clinical metric in identifying heart failure patients as potential responders to CRT. Electromechanical models of the heart have also been used as test beds to understand how different pacing parameters affect CRT efficacy $[59,60]$. Constantino et al. [54] recently proposed a strategy to optimize the response to CRT that involves placing the LV pacing electrode at a location that targets the regions with the longest electromechanical delay; maximal hemodynamic benefit occurred when the LV pacing site was located near the base and midventricle, which was within the region of longest electromechanical delay (Figures 4(a), $4(\mathrm{~b})$, and 4(c)). Optimization based on ATP consumption was also considered (Figures 4(d) and 4(e)). Niederer et al. [61] examined whether multisite CRT with a quadripolar lead could result in improved CRT response in a model of human electromechanics; it was found that that multisite CRT conferred a greater hemodynamic improvement only in infarcted hearts. Finally, patient-specific models of hearts with contractile dyssynchrony have been recently developed $[62,63]$, holding high promise to become an important clinical tool in the treatment of dyssynchronous heart failure.

With the advancements of computational techniques and medical imaging and image processing, the groundwork for patient-specific modeling of electromechanics has also been laid. Similar to electrophysiological applications as described above, the first step in conducting patient-specific cardiac electromechanical simulations is to construct the computational model of the heart from the patient's medical images. In addition to the challenges in reconstructing heart geometry from in vivo clinical scans of low resolution for any other applications, a specific hurdle in constructing the geometric mesh of a patient's heart for electromechanical applications is defining the unloaded state of the heart, since the heart is constantly loaded during image acquisition. Aguado-Sierra et al. [62] used an iterative estimation scheme to approximate the unloaded geometry from the end-diastolic geometry and ventricular pressures. To help accelerate the clinical adoption of personalized simulations, Lamata et al. [64] developed a robust method that uses variation warping technique to accurately and quickly construct patient-specific meshes from a template heart in a matter of minutes. Equally as important is parameterizing and enriching the electromechanical model of the heart with the patient's own clinical data, as done in studies by Sermesant et al. [63] and Aguado-Sierra et al. [62] These initial results in the development of patient-specific cardiac electromechanical models attest to the potential utility of cardiac simulation in the diagnosis and treatment of disorders in the pumping function of the heart.

\section{Computational Modeling of Cardiac Defibrillation}

Controlling the complex spatiotemporal dynamics underlying life-threatening cardiac arrhythmias such as fibrillation is extremely difficult because of the nonlinear interaction of excitation waves in a heterogeneous anatomical substrate. In the absence of a better strategy, strong electrical shocks have remained the only reliable treatment for cardiac fibrillation. Over the years, biophysically detailed multiscale models of defibrillation have made major contributions to understanding how defibrillation shocks used in clinical practice interact 


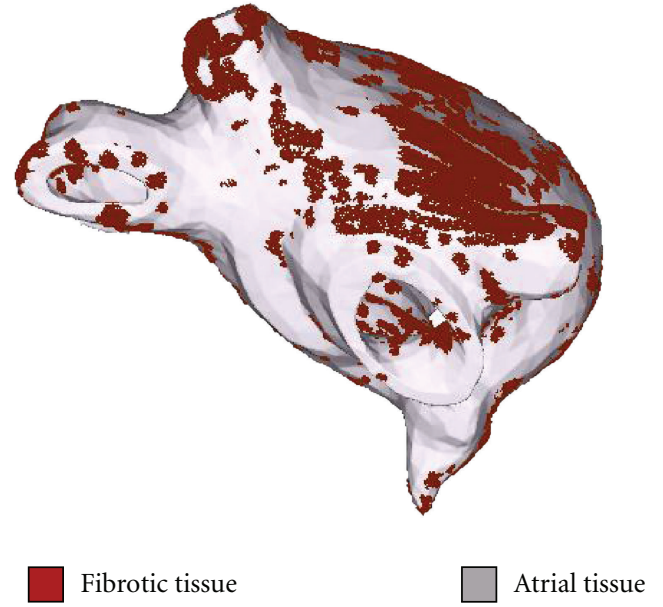

(a)

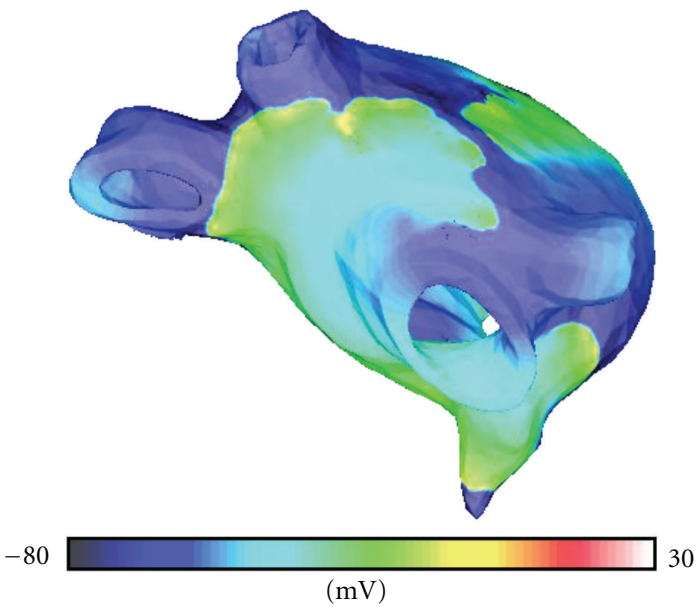

(b)

FIGURE 3: Model of human left atrium with fibrosis (a) and atrial fibrillation in the model (showing transmembrane potential distribution, (b)). Arrhythmia is induced following pulmonary vein ectopy. Figure modified from [53].

with cardiac tissue [67-76]. Computer modeling of wholeheart defibrillation has been instrumental in the development of the virtual electrode polarization (VEP) theory for defibrillation. Research has found that mechanisms for shock success or failure are multifactorial, depending mainly on the postshock distribution of transmembrane potential as well as timing and speed of propagation of the shock-induced wavefronts. Recent simulation studies have been instrumental in understanding the mechanisms for the existence of the isoelectric window following defibrillation shocks of strength near the defibrillation threshold (DFT); the isoelectric window is due to propagation of the postshock activations through intramural excitable areas ("tunnel propagation"), bounded by the long-lasting postshock depolarization of the surfaces $[65,77]$. Figure 5 presents a simulation of tunnel propagation following the delivery of a defibrillating shock to the fibrillating rabbit ventricles. Simulations have also contributed to understanding of the process of defibrillation in hears with myocardial ischemia and infarction [78-80]. Finally, using a rabbit ventricular electromechanics model, Trayanova et al. [81] conducted simulations of vulnerability to strong shocks and defibrillation under the conditions of $\mathrm{LV}$ dilation and determined the mechanisms by which mechanical deformation may lead to increased vulnerability and elevated DFT. The results of the study suggested that ventricular geometry and the rearrangement of fiber architecture in the deformed ventricles are responsible for the reduced defibrillation efficacy in the dilated ventricles.

Recently, defibrillation modeling has focused on the development of new methodologies for low-voltage termination of lethal arrhythmias or for applying defibrillation in novel, less damaging ways. The study by Tandri et al. [82] was based on the premise that sustained kilohertzrange alternating current $(\mathrm{AC})$ fields have been known to instantaneously and reversibly block electrical conduction in nerve tissue. The article provided proof of the concept that electric fields, such as those used for neural block, when applied to cardiac tissue, similarly produce reversible block of cardiac impulse propagation and lead to successful defibrillation, and that this methodology could potentially be safer means for termination of life-threatening reentrant arrhythmias. The data revealed a previously unrecognized capacity for myocardial cells to be placed in an extended, yet immediately reversible, state of refractoriness by an applied electric field. The imposed refractory state blocked all wave propagation and resulted in termination of reentrant arrhythmias, without impairment of subsequent cellular electrical function or initiation of postshock fibrillatory activity. Since the same AC fields block equally well both neural and cardiac activity, the proposed defibrillation methodology could possibly be utilized to achieve highvoltage yet painless defibrillation.

\section{Computational Modeling of the Heart as a Testbed for New Molecular Therapies}

A major avenue of scientific inquiry in computational cardiology relates the binding/unbinding of drugs to molecular target(s) to the instigation, termination or prevention of cardiac arrhythmias. In this section we focus on examples where emergent behavior, resulting from integrations across the scales of biological hierarchy, has shed new light on existing or novel drug actions for treatment of arrhythmia.

At the level of the ion channel, Markov models with state specific drug binding/unbinding have been used to test hypotheses regarding the mechanisms of drug effects on macroscopic currents. Since the arrhythmogenic long QT syndrome (LQTS) type 2 is characterized by loss of repolarizing rapid delayed rectifier $\mathrm{K}^{+}$current, $I_{\mathrm{Kr}}$ [83], a straightforward approach for prevention of LQT-2 arrhythmia, therefore, would be pharmacological enhancement of $I_{\mathrm{Kr}}$. Perry et al. [84] explored the novel compound RPR260243, shown to enhance $I_{\mathrm{Kr}}$, represented by the KCNH2 isoform 1a current expressed in xenopus oocytes. Rate constants 


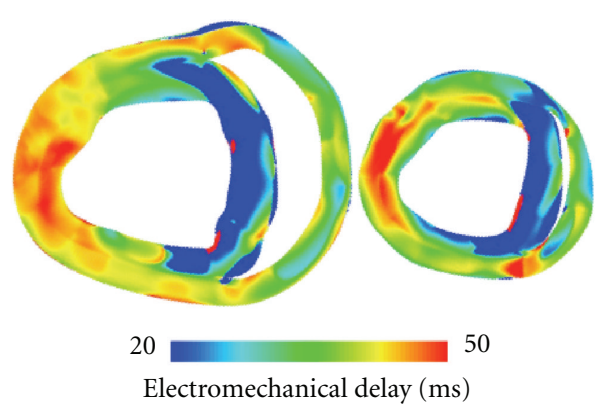

(a)

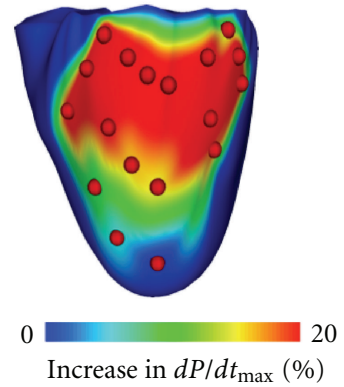

(b)

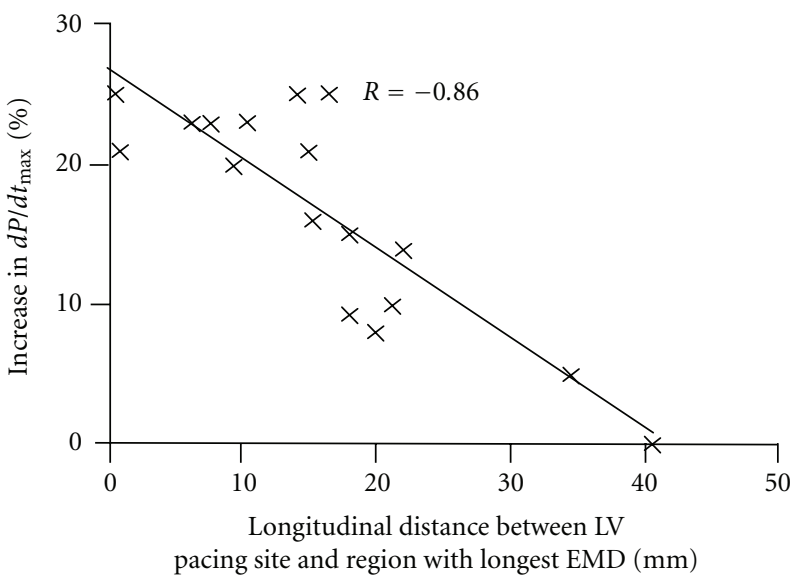

(c)

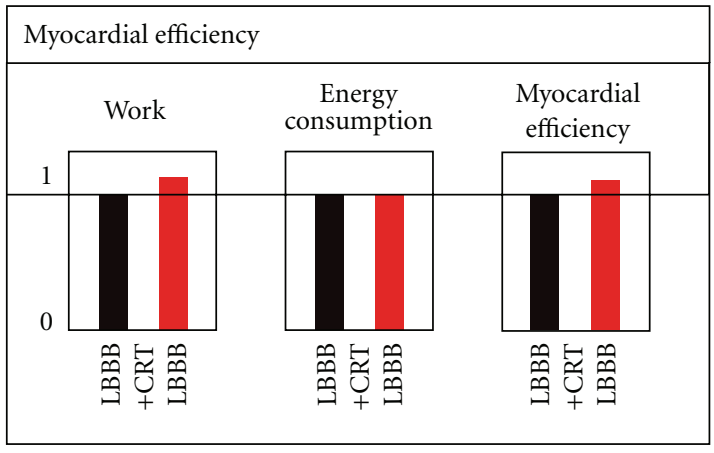

(d)

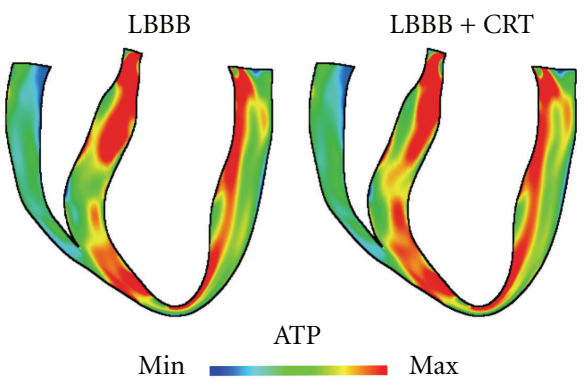

(e)

FIGURE 4: Using electromechanical models of dyssynchronous heart failure to optimize CRT response. (a) Transmural short-axis EMD maps during left bundle branch block (LBBB). (b) Map of the percentage increase in dP/dtmax as a function of the LV pacing site. Red dots denote LV pacing sites. (c) Correlation of longitudinal distance between LV pacing site and region with longest EMD, and percentage increase in $\mathrm{dP} / \mathrm{dtmax}$. (d) Bar graph of stroke work (left), total ventricular energy consumption (middle), and myocardial efficiency (right) during LBBB and following CRT. Values are normalized to LBBB (baseline) values. (e) Distribution of ATP consumption during LBBB and following CRT. Figure modified from [54].

in a proposed modal gating scheme were determined to best fit the experimental data. The model revealed that $I_{\mathrm{Kr}}$ enhancement could be explained by dose-dependent loss of deactivation; point mutation analysis provided the structural mechanism behind this model prediction. Going a step further, Sale et al. [85] simulated the effects of the drug E4031, known to block $I_{\mathrm{Kr}}$ in its open state in a use dependent manner [86] on the human ventricular action potential. Drug E4031 is known to block $I_{\mathrm{Kr}}$ in its open state, in a use-dependent manner [86]. Previously it was unclear why isoform 1a current, which is smaller than $1 \mathrm{a} / 1 \mathrm{~b}$ combination, was more sensitive to E4031. The Markov model for 1a alone versus $1 \mathrm{a} / 1 \mathrm{~b}$ mechanistically explained the discrepancy, and related action potential prolongation with E4031 as seen experimentally.

Type 3 LQTS is brought on by enhancement of the noninactivating, or late $\mathrm{Na}^{+}$current $\left(I_{\mathrm{Na}}\right)$ [83]. Using Markov models, Clancy et al. [87] compared the effect of 


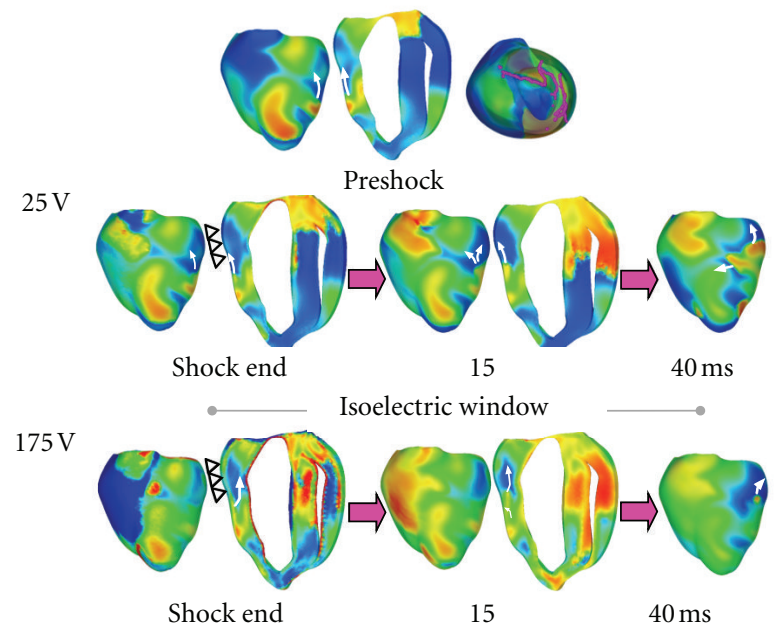

FIGURE 5: Tunnel propagation of activation following defibrillation shocks in the rabbit heart. Arrows indicate direction of propagation. Presented is the sub- merging of a preshock fibrillatory wavefront by a strong biphasic shock delivered from an ICD. Figure shows the model, the fibrillatory preshock state (with scroll-wave filaments, the organizing centers of reentry, shown in pink), and postshock transmembrane potential maps for two shock strengths at different postshock timings. In contrast to the 25-V shock, the near-DFT 175-V shock converted the left ventricular (LV) excitable area into an intramural excitable tunnel (see triangular arrows in shock-end panel) with no apparent propagation on the epicardium; the wavefront propagated in it until epicardial breakthrough following the isoelectric window. Images based on figures published in [65].

two $I_{\mathrm{Na}}$ blocking drugs, lidocaine and mexiletine, revealing that mexiletine preferentially binds to the population of channels undergoing late burst opening; the latter events are dangerously common in LQT3, leading to arrhythmogenic early afterdepolarizations (EADs). By contrast, lidocaine preferentially binds to channels during the rapid activation/inactivation phase of $I_{\mathrm{Na}}$. The model showed that there are mexiletine doses, which selectively remove late current and EADs without detrimental effect to excitability.

Nesterenko et al. [88] also drew connections between drug binding kinetics and emergent effects on the AP in the investigation of the novel antiarrhythmic drug ranolazine. Among its many targets [89], ranolazine reduces late $I_{\mathrm{Na}}$ in atrial-selective fashion [90]; Nesterenko et al. explained the mechanism behind this selectivity by introducing the concept of a "pre-open" state to the $I_{\mathrm{Na}}$ Markov model. The effect of late $I_{\mathrm{Na}}$ block by ranolazine on tissue electrophysiology was examined by Morita et al. [91], who demonstrated that late $I_{\mathrm{Na}}$ block suppresses EADs that lead to focal reentry after hydrogen peroxide application, as observed in experiments. Importantly, ranolazine is a mild $I_{\mathrm{Kr}}$ blocker at clinical doses, an effect of major significance for drug safety. Since current FDA regulations require that new candidate drugs not block $I_{\mathrm{Kr}}$, ranolazine would not have been approved if discovered today. Rodriguez et al. [92] proposed that computer modeling of multichannel affecting drugs, such as ranolazine, could be a testbed for determining the utility of new or previously rejected compounds or drug combination approaches, with modeling as a force against rigid standards, and toward rational, more holistic drug candidate selection. Fundamentally, this is the modeling approach of Sarkar and Sobie [93] whose recent article explores basic mechanisms by which interrelated model parameters contribute to the consequence of $I_{\mathrm{Kr}}$ block, a phenomenon known as the "repolarization reserve" [94]. The article makes the important discovery that subtle changes in ion channel substrate can have profound and indirect effects on the response to drugs. Simulating the subtle differences between species [95] and the effects of sex hormones $[96,97]$ also demonstrated changes in drug block effects. Personalized medicine requires clear delineation of the subtle interspecies and interindividual differences, which determine outcomes; this delineation is made possible in part by mechanistic simulation.

Relating effects of drugs on ion channels beyond the AP require virtual tissue or whole heart organ simulation, to examine arrhythmia onset, termination, and prevention. Recently, Benson et al. [98] related the effects of d-sotalol, an $I_{\mathrm{Kr}}$ blocker, and amiodarone, a complex multichannel effector, to arrhythmia formation in the heterogenous canine ventricular wedge. An emergent finding was the understanding of how the vulnerability window is enhanced by $\mathrm{d}$-sotalol but reduced by amiodarone due to different effects of the drugs on different cell types. Whereas the drug models used by Benson et al. were implemented by simple conduction scaling, a new study by Moreno et al. has incorporated both state-dependent Markov modeling of drug effects and full integration to the human AP, human tissue, and finally realistic MRI image-based human heart [66]. This is the first instance of such massive integration across the space and time scales at play. Moreno et al. showed that the effects of flecainide and lidocaine on $I_{\mathrm{Na}}$ block are globally similar in response to dynamic protocols. However, clinical trials have 


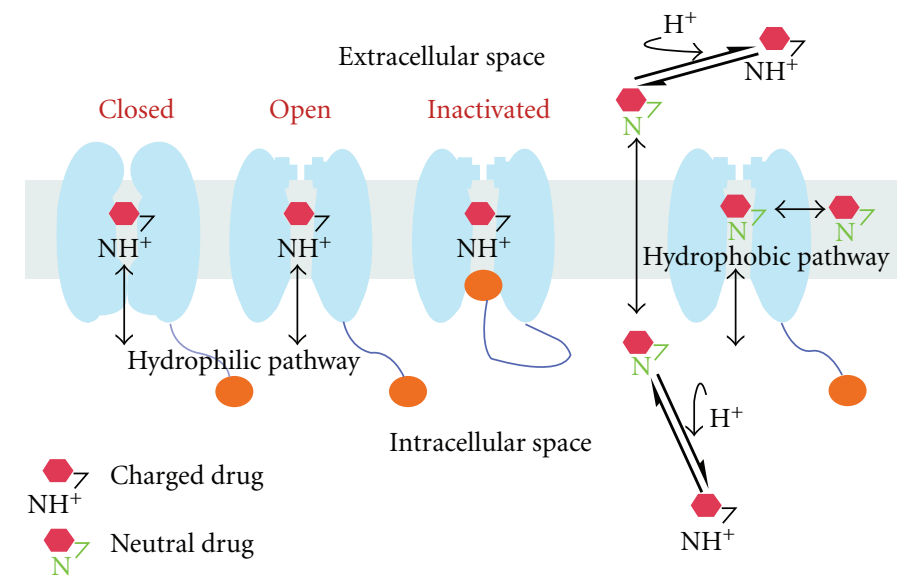

(a) Model schematic

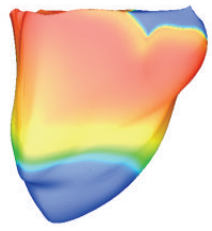

$500 \mathrm{~ms}$

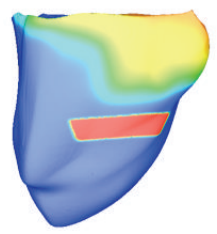

$720 \mathrm{~ms}$

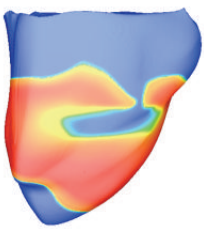

$920 \mathrm{~ms}$

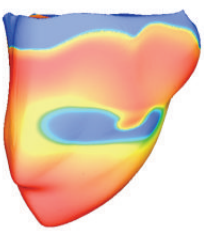

$1020 \mathrm{~ms}$

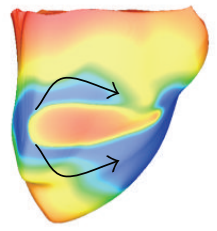

$1195 \mathrm{~ms}$

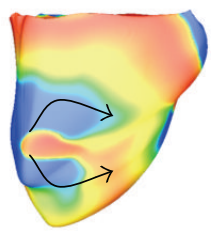

$3500 \mathrm{~ms}$

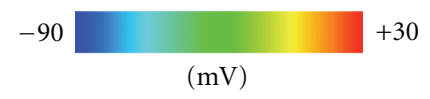

(b) Flecainide

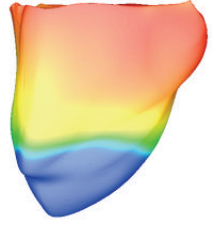

$500 \mathrm{~ms}$

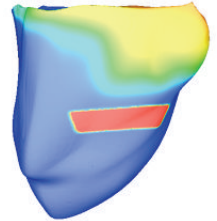

$670 \mathrm{~ms}$

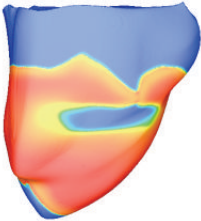

$870 \mathrm{~ms}$

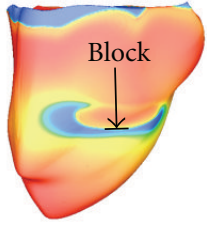

$970 \mathrm{~ms}$

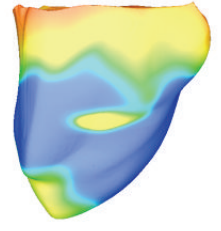

$1170 \mathrm{~ms}$

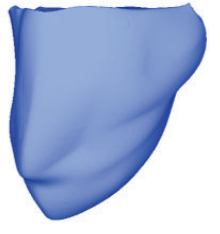

$3500 \mathrm{~ms}$

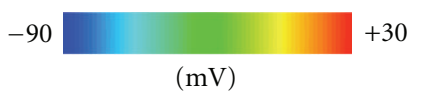

(c) Lidocaine

FIGURE 6: Simulation of drug-related arrhythmias. (a) Schematic for drug binding to sodium channels. Maps of the phase variable in (b), sustained figure-of-eight reentry with $2 \mu \mathrm{M}$ flecainide, and (c) nonsustained reentry with $20 \mu \mathrm{M}$ lidocaine, following premature stimuli (S2). Sustained reentry occurred when applying S2 within the vulnerable window (VW) of the model with $2 \mu \mathrm{M}$ flecainide (VW $=660 \mathrm{~ms}-$ $735 \mathrm{~ms}$ ), but not for the model with $20 \mu \mathrm{M}$ lidocaine (VW $=630 \mathrm{~ms}-685 \mathrm{~ms}$ ), as indicated by the $970 \mathrm{~ms}$ time point of (c). Figure modified from [66].

shown previously that flecainide tended to be proarrhythmic at therapeutic doses, while lidocaine was not. Moreno et al. results make clear that neither simple reduction in sodium conductance nor single cell simulation can resolve this paradox. At the macroscopic scale, the vulnerable window was greater for flecainide than for lidocaine (especially in heart failure simulations due to shortened diastole) and reentrant arrhythmia in the ventricle persisted (Figure 6). At the microscopic scale, Markov models explained that this was due to the relatively slow accumulation of and recovery from use dependent block with flecainide.

\section{Noninvasive Electroanatomic Mapping of the Heart}

The electrocardiogram (ECG), a century-old tool for assessing the electrical activity of the heart, is a global manifestation, on the body surface, of the electrical activity that takes place in the heart during the cardiac cycle. The electrical sources within the heart, separated by tissues from the body surface, are sampled on the body surface by only a few electrodes. This poses a challenge in the treatment of lethal ventricular arrhythmias. Thus, clinicians have sought 
to gain insight into the spatiotemporal patterns of electrical activation in the heart by interpreting other noninvasively recorded signals. Inverse electrocardiography represents the development on noninvasive methodology for assessing the spatiotemporal electrical activation in the heart, in which body surface electrograms and patient-specific anatomical data are combined with state-of-the-art computational techniques. From a mathematical standpoint, the reconstruction of signals in the domain of the heart from body surface measurements is ill-posed, in that it can easily be corrupted by low-amplitude electric noise or minute positional errors. Thus, the development of tools that can be implemented in clinical practice has been a long and arduous road.

The application of inverse electrocardiography in humans has been led by the Rudy lab, whose electrocardiographic imaging (ECGI) method computes epicardial extracellular potential distributions from body surface potential maps. ECGI has been performed on WolffParkinson-White (WPW) patients before and after accessory pathway ablation [99], to characterize the size and extent of scar in postmyocardial infarction patients [100], to identify responders to CRT therapy [101], to noninvasively map infarct-related ventricular arrhythmias [102], and to examine the spontaneous initiation of ventricular tachycardia (VT) and its termination with antitachycardia pacing. The technique was also used in a wide variety of AF patients and has demonstrated that multiple $\mathrm{AF}$ mechanisms (isthmus propagation, macroscopic reentry, multiple coexisting wavelets, and wave break) often occur simultaneously in the same patient [103].

Studies by the teams of $\mathrm{He}$, Oosterom, Berger, and Kalinin [104-108] have advanced noninvasive electrocardiographic imaging beyond the mapping of epicardial electrograms to mapping intramural or endocardial electrical activity. The inverse methodology by the He team reconstructs an equivalent current density field throughout the ventricles, from which $3 \mathrm{D}$ maps of activation sequence are generated; while it has not been yet utilized in patient studies, the technique was able to accurately pinpoint sources of endocardial ectopy in pigs [109] and to image activation sequences during pacing and VT in both rabbits [110] and dogs [111]. Kalinin's approach $[108,112]$ to map epicardial and endocardial electrograms has been already applied in 200 patients in Russian hospitals [113]. Similarly, the methodology developed by Berger and coworkers has applied to identify preexcitation sites in WPW patients [107] and to provide detail on simultaneous endocardial and epicardial activation sequences during CRT [105].

\section{Biophysically Detailed Computational Modeling of the Heart in Risk Stratification for Arrhythmias}

Robust methods for stratifying the risk of lethal cardiac arrhythmias decrease morbidity and mortality in patients with cardiovascular disease and reduce health care costs [114]. The most widely used approaches currently used for stratifying risk of cardiac arrhythmias involve testing for abnormalities in the ECG, then using the results to identify patients who would benefit from ICD therapy. ECGbased risk stratification methods scan for abnormalities in ventricular depolarization (late potentials [115] and a fractionated QRS complex [116]) and repolarization (Twave alternans [117], QT variability or dispersion [118, 119]). However, the mechanisms underlying these ECG indices, and their relationship to lethal cardiac arrhythmias, are not fully understood. This lack of knowledge likely explains why results of clinical trials to correlate surface EGG indices to lethal cardiac arrhythmias are often contradictory [114]. Computational models of the heart have made inroads in this clinical cardiology arena [98, 120-126].

Research has reported a strong correlation between increased arrhythmia risk and the presence of T-wave alternans $[127,128]$. In the clinical setting, testing for Microvolt T-wave Alternans (MTWA) has particularly shown promise for dichotomizing patients that would and would not benefit from ICD therapy $[129,130]$. However, the mechanistic basis of MTWA preceding lethal ventricular arrhythmias has been under debate. Until recently, it was believed that a steep action potential duration $(\mathrm{APD})$ restitution $(>1)$ at rapid heart rates [131] produces alternans in APD that underlie Twave alternans and the genesis of fibrillation [132]. However, MTWA is most successful in stratifying risk in patients at heart rates $<110 \mathrm{bpm}$, where APD restitution is flat [133]. Computational models of the LV wall in combination with clinical data revealed that abnormal handing of intracellular calcium underlies alternans in action potential voltage, which result in MTWA at heart rates $<110 \mathrm{bpm}[120$, 121]; abnormalities in intracellular calcium have long been linked to ventricular fibrillation $[134,135]$. Computational modeling studies have also shown that under the conditions of abnormal calcium dynamics, the magnitude of the T-wave alternans is enhanced by structural heterogeneities in the myocardium [122].

A recent study used MRI-based computational model of the human ventricles to demonstrate that detecting instabilities in the QT interval in the clinical ECGs can predict the onset of VT, particularly in patients with acute myocardial infarction [126]. By having the ability to easily control the frequency and degree of premature activations in the model, the studies found that increased frequency of premature activation can precede the onset of VT, with the premature activations placing the system in a state where QT interval is unstable. Therefore, screening the QT interval of the ECG for instabilities using the novel algorithm developed by Chen and Trayanova $[126,136]$ could potentially be a robust risk stratification method for patients with acute myocardial infarction. These study pave the way for executing computer simulations to determine patient-specific thresholds for arrhythmia stratification ECG indices, rather than relaying on clinical guidelines based on large and diverse cohorts of patients. Another approach for stratifying the risk of lethal cardiac arrhythmias that has recently gained traction is the use of computer models to predict the arrhythmia outcome in patients that exhibit potentially lethal mutations in genes encoding cardiac proteins associated with the long-QT syndrome $[98,123-125]$. These studies chart 
new directions for future genotype-based risk stratification and personalized gene therapy.

\section{Final Words}

Modern cardiac research has increasingly recognized that computational models of the heart can help interpret an array of experimental data and dissect important mechanisms and relationships. This paper focused on another aspect of computational modeling of the heart: its translational potential. Computer simulations of the function of the diseased heart represent a profound example of a research avenue in the new discipline of computational medicine [29], aiming specifically at improving the clinical practice of cardiology. Biophysically detailed models of the heart assembled with data from cardiac imaging modalities that incorporate electromechanical and structural remodeling in cardiac disease are well poised to become a first line of screening of new therapies and approaches, new diagnostic developments, and new methods for disease prevention. Implementing patient-specific cardiac simulations at the patient bedsides could become one of the most thrilling examples of multidisciplinary approaches in translational medicine.

\section{Acknowledgments}

This research was supported by NIH R01 award HL103428, HL105216, and HL094610 and NSF awards CBET-0933029 and 1124804 .

\section{References}

[1] D. Noble, "Modeling the heart-from genes to cells to the whole organ," Science, vol. 295, no. 5560, pp. 1678-1682, 2002.

[2] N. A. Trayanova, "Whole-heart modeling : applications to cardiac electrophysiology and electromechanics," Circulation Research, vol. 108, no. 1, pp. 113-128, 2011.

[3] E. Vigmond, F. Vadakkumpadan, V. Gurev et al., "Towards predictive modelling of the electrophysiology of the heart," Experimental Physiology, vol. 94, no. 5, pp. 563-577, 2009.

[4] R. H. Clayton, "Vortex filament dynamics in computational models of ventricular fibrillation in the heart," Chaos, vol. 18, no. 4, Article ID 043127, 12 pages, 2008.

[5] K. H. W. J. ten Tusscher, R. Hren, and A. V. Panfilov, "Organization of ventricular fibrillation in the human heart," Circulation Research, vol. 100, no. 12, pp. e87-e101, 2007.

[6] A. Garfinkel, Y. H. Kim, O. Voroshilovsky et al., "Preventing ventricular fibrillation by flattening cardiac restitution," Proceedings of the National Academy of Sciences of the United States of America, vol. 97, no. 11, pp. 6061-6066, 2000.

[7] O. Bernus, B. van Eyck, H. Verschelde, and A. V. Panfilov, "Transition from ventricular fibrillation to ventricular tachycardia: a simulation study on the role of $\mathrm{Ca}^{2+}$-channel blockers in human ventricular tissue," Physics in Medicine and Biology, vol. 47, no. 23, pp. 4167-4179, 2002.

[8] B. Echebarria and A. Karma, "Mechanisms for initiation of cardiac discordant alternans," European Physical Journal, vol. 146, no. 1, pp. 217-231, 2007.
[9] E. M. Cherry and F. H. Fenton, "Suppression of alternans and conduction blocks despite steep APD restitution: electrotonic, memory, and conduction velocity restitution effects," American Journal of Physiology, vol. 286, no. 6, pp. H2332H2341, 2004.

[10] R. H. Keldermann, K. H. W. J. ten Tusscher, M. P. Nash et al., "A computational study of mother rotor VF in the human ventricles," American Journal of Physiology, vol. 296, no. 2, pp. H370-H379, 2009.

[11] R. H. Keldermann, K. H. W. J. ten Tusscher, M. P. Nash, R. Hren, P. Taggart, and A. V. Panfilov, "Effect of heterogeneous APD restitution on VF organization in a model of the human ventricles," American Journal of Physiology, vol. 294, no. 2, pp. H764-H774, 2008.

[12] K. S. McDowell, H. J. Arevalo, M. M. Maleckar, and N. A. Trayanova, "Susceptibility to arrhythmia in the infarcted heart depends on myofibroblast density," Biophysical Journal, vol. 101, no. 6, pp. 1307-1315, 2011.

[13] R. Bordas, K. Gillow, Q. Lou et al., "Rabbit-specific ventricular model of cardiac electrophysiological function including specialized conduction system," Progress in Biophysics and Molecular Biology, vol. 107, no. 1, pp. 90-100, 2011.

[14] M. Deo, P. M. Boyle, A. M. Kim, and E. J. Vigmond, "Arrhythmogenesis by single ectopic beats originating in the Purkinje system," American Journal of Physiology, vol. 299, no. 4, pp. H1002-H1011, 2010.

[15] X. Jie and N. A. Trayanova, "Mechanisms for initiation of reentry in acute regional ischemia phase 1B," Heart Rhythm, vol. 7, no. 3, pp. 379-386, 2010.

[16] X. Jie, B. Rodríguez, J. R. de Groot, R. Coronel, and N. Trayanova, "Reentry in survived subepicardium coupled to depolarized and inexcitable midmyocardium: insights into arrhythmogenesis in ischemia phase 1B," Heart Rhythm, vol. 5, no. 7, pp. 1036-1044, 2008.

[17] X. Jie, V. Gurev, and N. Trayanova, "Mechanisms of mechanically induced spontaneous arrhythmias in acute regional ischemia," Circulation Research, vol. 106, no. 1, pp. 185-192, 2010.

[18] F. Vadakkumpadan, H. Arevalo, A. J. Prassl et al., "Imagebased models of cardiac structure in health and disease," Wiley Interdisciplinary Reviews, vol. 2, no. 4, pp. 489-506, 2010.

[19] M. J. Bishop, G. Plank, R. A. B. Burton et al., "Development of an anatomically detailed MRI-derived rabbit ventricular model and assessment of its impact on simulations of electrophysiological function," American Journal of Physiology, vol. 298, no. 2, pp. H699-H718, 2010.

[20] W. G. Stevenson, P. Brugada, and B. Waldecker, "Clinical, angiographic, and electrophysiologic findings in patients with aborted sudden death as compared with patients with sustained ventricular tachycardia after myocardial infarction," Circulation, vol. 71, no. 6, pp. 1146-1152, 1985.

[21] M. Pop, M. Sermesant, T. Mansi, E. Crystal, S. Ghate, J. Peyrat et al., "Correspondence between simple 3-D MRI-based computer models and in-vivo EP measurements in swine with chronic infarctions," IEEE Transactions on Biomedical Engineering, vol. 58, no. 12, pp. 3483-3486, 2011.

[22] H. Arevalo, G. Plank, P. Helm, H. Halperin, and N. Trayanova, "Volume of peri-infarct zone determines arrhythmogenesis in infarcted heart," Heart Rhythm, vol. 6, no. 5, pp. S232-S233, 2009.

[23] H. Arevalo, H. Estner, C. Park, H. Halperin, and N. Trayanova, "In-vivo MRI-based models of infarct- related 
ventricular tachycardia successfully predict optimal ablation site," Heart Rhythm, vol. 9, no. 5, p. S181, 2012.

[24] H. Ashikaga, H. Arevalo, F. Vadakkumpadan, R. Blake, R. Berger, H. Calkins et al., "MRI-based patient-specific virtual electrophysiology laboratory for scar-related ventricular tachycardia," Circulation, vol. 124, p. A541, 2011.

[25] J. Ng, J. T. Jacobson, J. K. Ng, D. Gordon, D. C. Lee, J. C. Carr et al., "Virtual electrophysiological study in a 3-dimensional cardiac magnetic resonance imaging model of porcine myocardial infarction," Journal of the American College of Cardiology, vol. 60, no. 5, pp. 423-430, 2012.

[26] J. Relan, P. Chinchapatnam, M. Sermesant, K. Rhode, M. Ginks, H. Delingette et al., "Coupled personalization of cardiac electrophysiology models for prediction of ischaemic ventricular tachycardia," Interface Focus, vol. 1, no. 3, pp. 396-407, 2011.

[27] F. Vadakkumpadan, H. Arevalo, C. Ceritoglu, M. Miller, and N. Trayanova, "Image-based estimation of ventricular fiber orientations for personalized modeling of cardiac electrophysiology," IEEE Transactions on Medical Imaging, vol. 31, no. 5, pp. 1051-1060, 2012.

[28] J. D. Bayer, R. C. Blake, G. Plank, and N. A. Trayanova, "A novel rule-based algorithm for assigning myocardial fiber orientation to computational heart models," Annals of Biomedical Engineering, vol. 40, no. 10, pp. 2243-2254, 2012.

[29] R. L. Winslow, N. Trayanova, D. Geman, and MI. Miller, "Computational medicine: translating models to clinical care," Science Translational Medicine, vol. 4, no. 158, p. 158rv11, 2012.

[30] N. Virag, V. Jacquemet, C. S. Henriquez et al., "Study of atrial arrhythmias in a computer model based on magnetic resonance images of human atria," Chaos, vol. 12, no. 3, pp. 754-763, 2002.

[31] E. S. Di Martino, C. Bellini, and D. S. Schwartzman, "In vivo porcine left atrial wall stress: computational model," Journal of Biomechanics, vol. 44, no. 15, pp. 2589-2594, 2011.

[32] G. Seemann, C. Höper, F. B. Sachse, O. Dössel, A. V. Holden, and H. Zhang, "Heterogeneous three-dimensional anatomical and electrophysiological model of human atria," Philosophical Transactions of the Royal Society A, vol. 364, no. 1843, pp. 1465-1481, 2006.

[33] J. Freudenberg, T. Schiemann, U. Tiede, and K. H. Höhne, "Simulation of cardiac excitation patterns in a threedimensional anatomical heart atlas," Computers in Biology and Medicine, vol. 30, no. 4, pp. 191-205, 2000.

[34] V. M. Spitzer and D. G. Whitlock, "The visible human dataset: the anatomical platform for human simulation," The Anatomical Record, vol. 253, no. 2, pp. 49-57, 1998.

[35] S. Kharche, C. J. Garratt, M. R. Boyett et al., "Atrial proarrhythmia due to increased inward rectifier current $\left(I_{K 1}\right)$ arising from KCNJ2 mutation-a simulation study," Progress in Biophysics and Molecular Biology, vol. 98, no. 2-3, pp. 186197, 2008.

[36] M. E. Ridler, M. Lee, D. McQueen, C. Peskin, and E. Vigmond, "Arrhythmogenic consequences of action potential duration gradients in the atria," Canadian Journal of Cardiology, vol. 27, no. 1, pp. 112-119, 2011.

[37] J. Kneller, R. Q. Zou, E. J. Vigmond, Z. G. Wang, L. J. Leon, and S. Nattel, "Cholinergic atrial fibrillation in a computer model of a two-dimensional sheet of canine atrial cells with realistic ionic properties," Circulation Research, vol. 90, no. 9, pp. E73-E87, 2002.
[38] V. Jacquemet, "Pacemaker activity resulting from the coupling with nonexcitable cells," Physical Review E, vol. 74, no. 1, part 1, Article ID 011908, 2006.

[39] N. Kuijpers, H. ten Eikelder, and S. Verheule, "Atrial anatomy influences onset and termination of atrial fibrillation: a computer model study," in Proceedings of the 5th International Conference on Functional Imaging and Modeling of the Heart (FIMH '09), vol. 5528 of Lecture Notes in Computer Science, pp. 285-294, Nice, France, June 2009.

[40] T. Krogh-Madsen, G. W. Abbott, and D. J. Christini, "Effects of electrical and structural remodeling on atrial fibrillation maintenance: a simulation study," PLOS Computational Biology, vol. 8, no. 2, Article ID e1002390, 2012.

[41] E. J. Vigmond, N. A. Trayanova, and R. A. Malkin, "Excitation of a cardiac muscle fiber by extracellularly applied sinusoidal current," Journal of Cardiovascular Electrophysiology, vol. 12, no. 10, pp. 1145-1153, 2001.

[42] E. J. Vigmond, V. Tsoi, S. Kuo et al., "The effect of vagally induced dispersion of action potential duration on atrial arrhythmogenesis," Heart Rhythm, vol. 1, no. 3, pp. 334-344, 2004.

[43] M. Rotter, L. Dang, V. Jacquemet, N. Virag, L. Kappenberger, and M. Haïssaguerre, "Impact of varying ablation patterns in a simulation model of persistent atrial fibrillation," Pacing and Clinical Electrophysiology, vol. 30, no. 3, pp. 314-321, 2007.

[44] P. Ruchat, L. Dang, J. Schlaepfer, N. Virag, L. K. von Segesser, and L. Kappenberger, "Use of a biophysical model of atrial fibrillation in the interpretation of the outcome of surgical ablation procedures," European Journal of Cardio-Thoracic Surgery, vol. 32, no. 1, pp. 90-95, 2007.

[45] P. Ruchat, L. Dang, N. Virag, J. Schlaepfer, L. K. von Segesser, and L. Kappenberger, "A biophysical model of atrial fibrillation to define the appropriate ablation pattern in modified maze," European Journal of Cardio-Thoracic Surgery, vol. 31, no. 1, pp. 65-69, 2007.

[46] V. Jacquemet, A. van Oosterom, J. M. Vesin, and L. Kappenberger, "Analysis of electrocardiograms during atrial fibrillation," IEEE Engineering in Medicine and Biology Magazine, vol. 25, no. 6, pp. 79-88, 2006.

[47] E. J. Vigmond and L. J. Leon, "Electrophysiological basis of mono-phasic action potential recordings," Medical and Biological Engineering and Computing, vol. 37, no. 3, pp. 359365, 1999.

[48] E. J. Vigmond, V. Tsoi, Y. Yin, P. Pagé, and A. Vinet, "Estimating atrial action potential duration from electrograms," IEEE Transactions on Biomedical Engineering, vol. 56, no. 5, pp. 1546-1555, 2009.

[49] V. Jacquemet and C. S. Henriquez, "Genesis of complex fractionated atrial electrograms in zones of slow conduction: a computer model of microfibrosis," Heart Rhythm, vol. 6, no. 6, pp. 803-810, 2009.

[50] M. W. Krueger, S. Severi, K. Rhode et al., "Alterations of atrial electrophysiology related to hemodialysis session: insights from a multiscale computer model," Journal of Electrocardiology, vol. 44, no. 2, pp. 176-183, 2011.

[51] K. M. Lim, S. B. Hong, J. W. Jeon, M. S. Gyung, B. H. Ko, S. K. Bae et al., "Predicting the optimal position and direction of a ubiquitous ECG using a multi-scale model of cardiac electrophysiology," in Proceedings of the Annual International Conference of the IEEE Engineering in Medicine and Biology Society (EMBC '11), pp. 993-996, Boston, Mass, USA, September 2011. 
[52] R. MacLeod, J. Blauer, E. Kholmovski, R. Ranjan, N. Marrouche, N. Trayanova et al., "Subject specific, image based analysis and modeling in patients with atrial fibrillation from MRI," in Proceedings of the 9th IEEE International Symposium on Biomedical Imaging (ISBI '12), ISBI Meeting Proceedings, p. 1364, Barcelona, Spain, May 2012.

[53] K. S. McDowell, F. Vadakkumpadan, R. C. Blake, J. Blauerb, G. Plank, R. S. MacLeod et al., "Methodology for patientspecific modeling of atrial fibrosis as a substrate for atrial fibrillation," Journal of Electrocardiology, vol. 45, no. 6, pp. 640-645, 2012.

[54] J. Constantino, Y. Hu, and N. A. Trayanova, "A computational approach to understanding the cardiac electromechanical activation sequence in the normal and failing heart, with translation to the clinical practice of CRT," Progress in Biophysics and Molecular Biology, vol. 110, no. 2-3, pp. 372379, 2012.

[55] V. Gurev, T. Lee, J. Constantino, H. Arevalo, and N. A. Trayanova, "Models of cardiac electromechanics based on individual hearts imaging data: image-based electromechanical models of the heart," Biomechanics and Modeling in Mechanobiology, vol. 10, no. 3, pp. 295-306, 2011.

[56] R. C. P. Kerckhoffs, A. D. McCulloch, J. H. Omens, and L. J. Mulligan, "Effect of pacing site and infarct location on regional mechanics and global hemodynamics in a model based study of heart failure," in Proceedings of the 4th International Conference on Functional Imaging and Modeling of the Heart (FIMH '07), vol. 4466 of Lecture Notes in Computer Science, pp. 350-360, June 2007.

[57] R. C. P. Kerckhoffs, A. D. McCulloch, J. H. Omens, and L. J. Mulligan, "Effects of biventricular pacing and scar size in a computational model of the failing heart with left bundle branch block," Medical Image Analysis, vol. 13, no. 2, pp. 362 369, 2009.

[58] S. A. Niederer, G. Plank, P. Chinchapatnam et al., "Lengthdependent tension in the failing heart and the efficacy of cardiac resynchronization therapy," Cardiovascular Research, vol. 89, no. 2, pp. 336-343, 2011.

[59] R. C. P. Kerckhoffs, J. Lumens, K. Vernooy et al., "Cardiac resynchronization: insight from experimental and computational models," Progress in Biophysics and Molecular Biology, vol. 97, no. 2-3, pp. 543-561, 2008.

[60] S. A. Niederer, A. K. Shetty, G. Plank, J. Bostock, R. Razavi, N. P. Smith et al., "Biophysical modeling to simulate the response to multisite left ventricular stimulation using a quadripolar pacing lead," Pacing and Clinical Electrophysiology, vol. 35, no. 2, pp. 204-214, 2011.

[61] S. A. Niederer, A. K. Shetty, G. Plank, J. Bostock, R. Razavi, N. P. Smith et al., "Biophysical modeling to simulate the response to multisite left ventricular stimulation using a quadripolar pacing lead," Pacing and Clinical Electrophysiology, vol. 35, no. 2, pp. 204-214, 2012.

[62] J. Aguado-Sierra, A. Krishnamurthy, C. Villongco, J. Chuang, E. Howard, M. J. Gonzales et al., "Patient-specific modeling of dyssynchronous heart failure: a case study," Progress in Biophysics and Molecular Biology, vol. 107, no. 1, pp. 147-155, 2011.

[63] M. Sermesant, R. Chabiniok, P. Chinchapatnam, T. Mansi, F. Billet, P. Moireau et al., "Patient-specific electromechanical models of the heart for the prediction of pacing acute effects in CRT: a preliminary clinical validation,” Medical Image Analysis, vol. 16, no. 1, pp. 201-215, 2012.

[64] P. Lamata, S. Niederer, D. Nordsletten et al., "An accurate, fast and robust method to generate patient-specific cubic
Hermite meshes," Medical Image Analysis, vol. 15, no. 6, pp. 801-813, 2011.

[65] J. Constantino, Y. Long, T. Ashihara, and N. A. Trayanova, "Tunnel propagation following defibrillation with ICD shocks: hidden postshock activations in the left ventricular wall underlie isoelectric window," Heart Rhythm, vol. 7, no. 7, pp. 953-961, 2010.

[66] J. D. Moreno, Z. I. Zhu, P. C. Yang, J. R. Bankston, M. T. Jeng, C. Kang et al., "A computational model to predict the effects of class I anti-arrhythmic drugs on ventricular rhythms," Science Translational Medicine, vol. 3, no. 98, p. 98ra83, 2011.

[67] C. Anderson and N. A. Trayanova, "Success and failure of biphasic shocks: results of bidomain simulations," Mathematical Biosciences, vol. 174, no. 2, pp. 91-109, 2001.

[68] H. Arevalo, B. Rodriguez, and N. Trayanova, "Arrhythmogenesis in the heart: multiscale modeling of the effects of defibrillation shocks and the role of electrophysiological heterogeneity," Chaos, vol. 17, no. 1, Article ID 015103, 13 pages, 2007.

[69] T. Ashihara and N. A. Trayanova, "Asymmetry in membrane responses to electric shocks: insights from bidomain simulations," Biophysical Journal, vol. 87, no. 4, pp. 2271-2282, 2004.

[70] D. W. Bourn, R. A. Gray, and N. A. Trayanova, "Characterization of the relationship between preshock state and virtual electrode polarization-induced propagated graded responses resulting in arrhythmia induction," Heart Rhythm, vol. 3, no. 5, pp. 583-595, 2006.

[71] E. Entcheva, N. A. Trayanova, and F. J. Claydon, "Patterns of and mechanisms for shock-induced polarization in the heart: a bidomain analysis," IEEE Transactions on Biomedical Engineering, vol. 46, no. 3, pp. 260-270, 1999.

[72] A. E. Lindblom, B. J. Roth, and N. A. Trayanova, "Role of virtual electrodes in arrhythmogenesis: pinwheel experiment revisited," Journal of Cardiovascular Electrophysiology, vol. 11, no. 3, pp. 274-285, 2000.

[73] B. Rodríguez, J. C. Eason, and N. Trayanova, "Differences between left and right ventricular anatomy determine the types of reentrant circuits induced by an external electric shock. A rabbit heart simulation study," Progress in Biophysics and Molecular Biology, vol. 90, no. 1-3, pp. 399-413, 2006.

[74] B. Rodríguez, L. Li, J. C. Eason, I. R. Efimov, and N. A. Trayanova, "Differences between left and right ventricular chamber geometry affect cardiac vulnerability to electric shocks," Circulation Research, vol. 97, no. 2, pp. 168-175, 2005.

[75] N. Trayanova, K. Skouibine, and P. Moore, "Virtual electrode effects in defibrillation," Progress in Biophysics and Molecular Biology, vol. 69, no. 2-3, pp. 387-403, 1998.

[76] N. Trayanova, J. Constantino, T. Ashihara, and G. Plank, "Modeling defibrillation of the heart: approaches and insights," IEEE Reviews in Biomedical Engineering, vol. 4, pp. 89-102, 2011.

[77] T. Ashihara, J. Constantino, and N. A. Trayanova, “Tunnel propagation of postshock activations as a hypothesis for fibrillation induction and isoelectric window," Circulation Research, vol. 102, no. 6, pp. 737-745, 2008.

[78] B. Rodríguez, B. Tice, R. Blake, D. Gavaghan, and N. Trayanova, "Vulnerability to electric shocks in the regionallyischemic ventricles," in Proceedings of the 28th Annual International Conference of the IEEE Engineering in Medicine and Biology Society (EMBS '06), vol. 1, pp. 2280-2283, New York, NY, USA, September 2006. 
[79] B. Rodríguez, B. M. Tice, J. C. Eason, F. Aguel, J. M. Ferrero Jr., and N. Trayanova, "Effect of acute global ischemia on the upper limit of vulnerability: a simulation study," American Journal of Physiology, vol. 286, no. 6, pp. H2078-H2088, 2004.

[80] L. J. Rantner, H. J. Arevalo, J. L. Constantino, I. R. Efimov, G. Plank, and N. A. Trayanova, "Three-dimensional mechanisms of increased vulnerability to electric shocks in myocardial infarction: altered virtual electrode polarizations and conduction delay in the peri-infarct zone," The Journal of Physiology, vol. 590, part 18, pp. 4537-4551, 2012.

[81] N. Trayanova, V. Gurev, J. Constantino, and Y. Hu, "Mathematical models of ventricular mechano-electric coupling and arrhythmia," in Cardiac Mechano-Electric Feedback and Arrhythmias, P. Kohl, F. Sachs, and M. R. Franz, Eds., pp. 258268, 2011.

[82] H. Tandri, S. H. Weinberg, K. C. Chang, R. Zhu, N. A. Trayanova, L. Tung et al., "Reversible cardiac conduction block and defibrillation with high-frequency electric field," Science Translational Medicine, vol. 3, no. 102, p. 102ra96, 2011.

[83] P. J. Schwartz, S. G. Priori, C. Spazzolini et al., "Genotypephenotype correlation in the long-QT syndrome: genespecific triggers for life-threatening arrhythmias," Circulation, vol. 103, no. 1, pp. 89-95, 2001.

[84] M. Perry, F. B. Sachse, and M. C. Sanguinetti, "Structural basis of action for a human ether-a-go-go-related gene 1 potassium channel activator," Proceedings of the National Academy of Sciences of the United States of America, vol. 104, no. 34, pp. 13827-13832, 2007.

[85] H. Sale, J. Wang, T. J. O’Hara et al., "Physiological properties of hERG $1 \mathrm{a} / 1 \mathrm{~b}$ heteromeric currents and a hERG $1 \mathrm{~b}$-specific mutation associated with long-QT syndrome," Circulation Research, vol. 103, no. 7, pp. e81-e95, 2008.

[86] P. S. Spector, M. E. Curran, M. T. Keating, and M. C. Sanguinetti, "Class III antiarrhythmic drugs block HERG, a human cardiac delayed rectifier $\mathrm{K}^{+}$channel open-channel block by methanesulfonanilides," Circulation Research, vol. 78, no. 3, pp. 499-503, 1996.

[87] C. E. Clancy, Z. I. Zhu, and Y. Rudy, "Pharmacogenetics and anti-arrhythmic drug therapy: a theoretical investigation," American Journal of Physiology, vol. 292, no. 1, pp. H66-H75, 2007.

[88] V. V. Nesterenko, A. C. Zygmunt, S. Rajamani, L. Belardinelli, and C. Antzelevitch, "Mechanisms of atrial-selective block of $\mathrm{Na}$ channels by ranolazine: II. Insights from a mathematical model," American Journal of Physiology, vol. 301, no. 4, pp. H1615-H1624, 2011.

[89] C. Antzelevitch, L. Belardinelli, A. C. Zygmunt et al., "Electrophysiological effects of ranolazine, a novel antianginal agent with antiarrhythmic properties," Circulation, vol. 110, no. 8, pp. 904-910, 2004.

[90] A. Burashnikov, J. M. Di Diego, A. C. Zygmunt, L. Belardinelli, and C. Antzelevitch, "Atrium-selective sodium channel block as a strategy for suppression of atrial fibrillation: differences in sodium channel inactivation between atria and ventricles and the role of ranolazine," Circulation, vol. 116, no. 13, pp. 1449-1457, 2007.

[91] N. Morita, J. H. Lee, Y. Xie et al., "Suppression of re-entrant and multifocal ventricular fibrillation by the late sodium current blocker ranolazine," Journal of the American College of Cardiology, vol. 57, no. 3, pp. 366-375, 2011.
[92] B. Rodriguez, K. Burrage, D. Gavaghan, V. Grau, P. Kohl, and D. Noble, "The systems biology approach to drug development: application to toxicity assessment of cardiac drugs," Clinical Pharmacology and Therapeutics, vol. 88, no. 1, pp. 130-134, 2010.

[93] A. X. Sarkar and E. A. Sobie, "Regression analysis for constraining free parameters in electrophysiological models of cardiac cells," PLoS Computational Biology, vol. 6, no. 9, Article ID e1000914, 2010.

[94] D. M. Roden and T. Yang, "Protecting the heart against arrhythmias: potassium current physiology and repolarization reserve," Circulation, vol. 112, no. 10, pp. 1376-1378, 2005.

[95] T. O’Hara and Y. Rudy, “Quantitative comparison of cardiac ventricular myocyte electrophysiology and response to drugs in human and nonhuman species," American Journal of Physiology, vol. 302, no. 5, pp. H1023-H1030, 2011.

[96] H. Nakamura, J. Kurokawa, C. X. Bai et al., "Progesterone regulates cardiac repolarization through a nongenomic pathway: an in vitro patch-clamp and computational modeling study," Circulation, vol. 116, no. 25, pp. 2913-2922, 2007.

[97] P. C. Yang, J. Kurokawa, T. Furukawa, and C. E. Clancy, "Acute effects of sex steroid hormones on susceptibility to cardiac arrhythmias: a simulation study," PLoS Computational Biology, vol. 6, no. 1, Article ID e1000658, 2010.

[98] A. P. Benson, M. Al-Owais, and A. V. Holden, "Quantitative prediction of the arrhythmogenic effects of de novo hERG mutations in computational models of human ventricular tissues," European Biophysics Journal, vol. 40, no. 5, pp. 627639, 2011.

[99] S. Ghosh, E. K. Rhee, J. N. Avari, P. K. Woodard, and Y. Rudy, "Cardiac memory in patients with Wolff-Parkinson-White syndrome: noninvasive imaging of activation and repolarization before and after catheter ablation," Circulation, vol. 118, no. 9, pp. 907-915, 2008.

[100] P. S. Cuculich, J. Zhang, Y. Wang, K. A. Desouza, R. Vijayakumar, P. K. Woodard et al., "The electrophysiological cardiac ventricular substrate in patients after myocardial infarction: noninvasive characterization with electrocardiographic imaging," Journal of the American College of Cardiology, vol. 58, no. 18, pp. 1893-1902, 2011.

[101] S. Ghosh, J. N. A. Silva, R. M. Canham et al., "Electrophysiologic substrate and intraventricular left ventricular dyssynchrony in nonischemic heart failure patients undergoing cardiac resynchronization therapy," Heart Rhythm, vol. 8, no. 5, pp. 692-699, 2011.

[102] Y. Wang, P. S. Cuculich, J. Zhang, K. A. Desouza, R. Vijayakumar, J. Chen et al., "Noninvasive electroanatomic mapping of human ventricular arrhythmias with electrocardiographic imaging," Science Translational Medicine, vol. 3, no. 98, p. 98ra84, 2011.

[103] P. S. Cuculich, Y. Wang, B. D. Lindsay et al., "Noninvasive characterization of epicardial activation in humans with diverse atrial fibrillation patterns," Circulation, vol. 122, no. 14, pp. 1364-1372, 2010.

[104] P. M. van Dam, T. F. Oostendorp, A. C. Linnenbank, and A. van Oosterom, "Non-invasive imaging of cardiac activation and recovery," Annals of Biomedical Engineering, vol. 37, no. 9, pp. 1739-1756, 2009.

[105] T. Berger, B. Pfeifer, F. F. Hanser et al., "Single-beat noninvasive imaging of ventricular endocardial and epicardial activation in patients undergoing CRT," PLoS ONE, vol. 6, no. 1, Article ID e16255, 2011. 
[106] C. Han, C. Liu, S. Pogwizd, and B. He, "Noninvasive threedimensional cardiac activation imaging on a rabbit model," in Proceedings of the Annual International Conference of the IEEE Engineering in Medicine and Biology Society (EMBC '09), pp. 3271-3273, September 2009.

[107] T. Berger, G. Fischer, B. Pfeifer et al., "Single-beat noninvasive imaging of cardiac electrophysiology of ventricular preexcitation," Journal of the American College of Cardiology, vol. 48, no. 10, pp. 2045-2052, 2006.

[108] A. V. Kalinin, "Iterative algorithm for the inverse problem of electrocardiography in a medium with piecewise-constant electrical conductivity," Computational Mathematics and Modeling, vol. 22, no. 1, pp. 30-34, 2011.

[109] D. Lai, C. Liu, M. D. Eggen, P. A. Iaizzo, and B. He, "Localization of endocardial ectopic activity by means of noninvasive endocardial surface current density reconstruction," Physics in Medicine and Biology, vol. 56, no. 13, pp. 4161-4176, 2011.

[110] C. Han, S. M. Pogwizd, C. R. Killingsworth, and B. He, "Noninvasive imaging of three-dimensional cardiac activation sequence during pacing and ventricular tachycardia," Heart Rhythm, vol. 8, no. 8, pp. 1266-1272, 2011.

[111] C. Han, S. M. Pogwizd, C. R. Killingsworth, and B. He, "Noninvasive reconstruction of the three-dimensional ventricular activation sequence during pacing and ventricular tachycardia in the canine heart," American Journal of Physiology, vol. 302, no. 1, pp. H244-H252, 2012.

[112] A. V. Kalinin, "Iterative algorithm for the inverse problem of electrocardiography in a medium with piecewise-constant electrical conductivity," Computational Mathematics and Modeling, vol. 22, no. 1, pp. 30-34, 2011.

[113] L. A. Bokeriia, A. S. Revishvili, A. V. Kalinin, V. V. Kalinin, O. A. Liadzhina, and E. A. Fetisova, "Hardware-software system for noninvasive electrocardiographic examination of heart based on inverse problem of electrocardiography," Meditsinskaia Tekhnika, no. 6, pp. 1-7, 2008.

[114] J. J. Goldberger, A. E. Buxton, M. Cain et al., "Risk stratification for arrhythmic sudden cardiac death: identifying the roadblocks," Circulation, vol. 123, no. 21, pp. 2423-2430, 2011.

[115] D. L. Kuchar, C. W. Thorburn, and N. L. Sammel, "Prediction of serious arrhythmic events after myocardial infarction: signal-averaged electrocardiogram, Holter monitoring and radionuclide ventriculography," Journal of the American College of Cardiology, vol. 9, no. 3, pp. 531-538, 1987.

[116] M. K. Das, B. Khan, S. Jacob, A. Kumar, and J. Mahenthiran, "Significance of a fragmented QRS complex versus a Q wave in patients with coronary artery disease," Circulation, vol. 113, no. 21, pp. 2495-2501, 2006.

[117] D. S. Rosenbaum, L. E. Jackson, J. M. Smith, H. Garan, J. N. Ruskin, and R. J. Cohen, "Electrical alternans and vulnerability to ventricular arrhythmias," The New England Journal of Medicine, vol. 330, no. 4, pp. 235-241, 1994.

[118] R. D. Berger, E. K. Kasper, K. L. Baughman, E. Marban, H. Calkins, and G. F. Tomaselli, "Beat-to-beat QT interval variability: novel evidence for repolarization lability in ischemic and nonischemic dilated cardiomyopathy," Circulation, vol. 96, no. 5, pp. 1557-1565, 1997.

[119] J. P. Couderc, W. Zareba, S. McNitt, P. Maison-Blanche, and A. J. Moss, "Repolarization variability in the risk stratification of MADIT II patients," Europace, vol. 9, no. 9, pp. 717-723, 2007.

[120] S. M. Narayan, J. D. Bayer, G. Lalani, and N. A. Trayanova, "Action potential dynamics explain arrhythmic vulnerability in human heart failure. A clinical and modeling study implicating abnormal calcium handling," Journal of the American College of Cardiology, vol. 52, no. 22, pp. 17821792, 2008.

[121] J. D. Bayer, S. M. Narayan, G. G. Lalani, and N. A. Trayanova, "Rate-dependent action potential alternans in human heart failure implicates abnormal intracellular calcium handling," Heart Rhythm, vol. 7, no. 8, pp. 1093-1101, 2010.

[122] A. N. Doshi and S. F. Idriss, "Effect of resistive barrier location on the relationship between T-wave alternans and cellular repolarization alternans: a 1-D modeling study," Journal of Electrocardiology, vol. 43, no. 6, pp. 566-571, 2010.

[123] J. T. Zhao, A. P. Hill, A. Varghese et al., "Not all hERG pore domain mutations have a severe phenotype: G584S has an inactivation gating defect with mild phenotype compared to G572S, which has a dominant negative trafficking defect and a severe phenotype," Journal of Cardiovascular Electrophysiology, vol. 20, no. 8, pp. 923-930, 2009.

[124] C. Jons, J. O-Uchi, A. J. Moss, M. Reumann, J. J. Rice, I. Goldenberg et al., "Use of mutant-specific ion channel characteristics for risk stratification of long QT syndrome patients," Science Translational Medicine, vol. 3, no. 76, p. 76ra28, 2011.

[125] T. O'Hara and Y. Rudy, “Arrhythmia formation in subclinical ("silent") long QT syndrome requires multiple insults: quantitative mechanistic study using the KCNQ1 mutation Q357R as example," Heart Rhythm, vol. 9, no. 2, pp. 275-282, 2012.

[126] X. Chen, Y. Hu, B. J. Fetics, R. D. Berger, and N. A. Trayanova, "Unstable QT interval dynamics precedes ventricular tachycardia onset in patients with acute myocardial infarction: a novel approach to detect instability in QT interval dynamics from clinical ECG," Circulation, vol. 4, no. 6, pp. 858-866, 2011.

[127] S. M. Narayan, "T-wave alternans and the susceptibility to ventricular arrhythmias," Journal of the American College of Cardiology, vol. 47, no. 2, pp. 269-281, 2006.

[128] Z. Qu, Y. Xie, A. Garfinkel, and J. N. Weiss, "T-wave alternans and arrhythmogenesis in cardiac diseases," Frontiers in Physiology, vol. 1, p. 154, 2010.

[129] D. M. Bloomfield, J. T. Bigger, R. C. Steinman et al., "Microvolt $\mathrm{T}$-wave alternans and the risk of death or sustained ventricular arrhythmias in patients with left ventricular dysfunction," Journal of the American College of Cardiology, vol. 47, no. 2, pp. 456-463, 2006.

[130] S. H. Hohnloser, T. Ikeda, and R. J. Cohen, "Evidence regarding clinical use of microvolt T-wave alternans," Heart Rhythm, vol. 6, no. 3, supplement, pp. S36-S44, 2009.

[131] J. N. Weiss, A. Karma, Y. Shiferaw, P. S. Chen, A. Garfinkel, and $\mathrm{Z} . \mathrm{Qu}$, "From pulsus to pulseless: the saga of cardiac alternans," Circulation Research, vol. 98, no. 10, pp. 12441253, 2006.

[132] J. M. Pastore, S. D. Girouard, K. R. Laurita, F. G. Akar, and D. S. Rosenbaum, "Mechanism linking T-wave alternans to the genesis of cardiac fibrillation," Circulation, vol. 99, no. 10, pp. 1385-1394, 1999.

[133] S. M. Narayan, M. R. Franz, G. Lalani, J. Kim, and A. Sastry, "T-wave alternans, restitution of human action potential duration, and outcome," Journal of the American College of Cardiology, vol. 50, no. 25, pp. 2385-2392, 2007.

[134] J. N. Weiss, M. Nivala, A. Garfinkel, and Z. Qu, "Alternans and arrhythmias : from cell to heart," Circulation Research, vol. 108, no. 1, pp. 98-112, 2011.

[135] F. M. Merchant and A. A. Armoundas, "Role of substrate and triggers in the genesis of cardiac alternans, from the myocyte 
to the whole heart: implications for therapy," Circulation, vol. 125, no. 3, pp. 539-549, 2012.

[136] X. Chen and N. A. Trayanova, "A novel methodology for assessing the bounded-input bounded-output instability in QT interval dynamics: application to clinical ECG with ventricular tachycardia," IEEE Transactions on Biomedical Engineering, vol. 59, no. 8, pp. 2111-2117, 2012. 


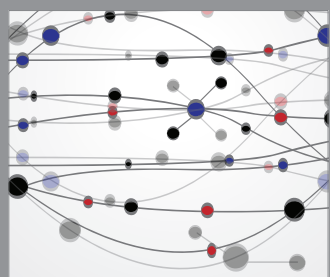

The Scientific World Journal
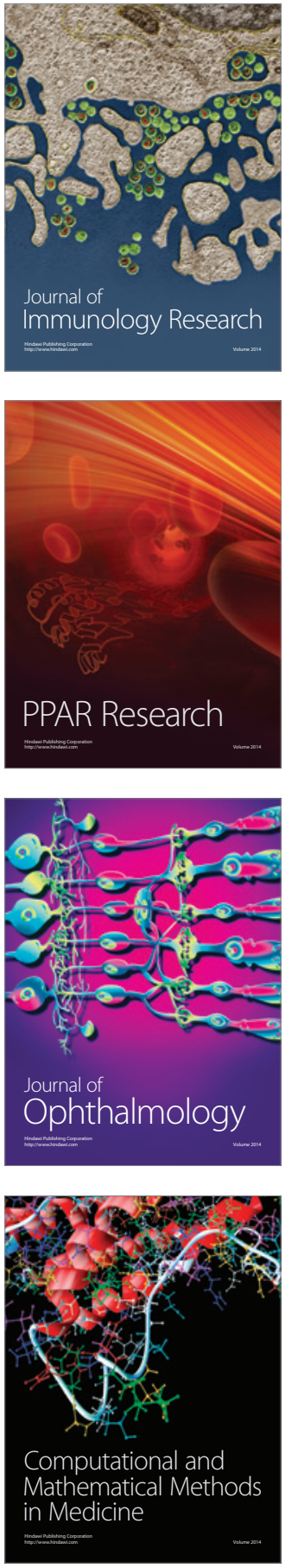

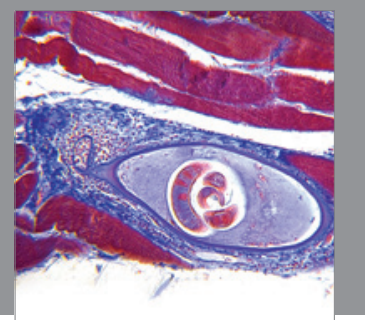

Gastroenterology

Research and Practice
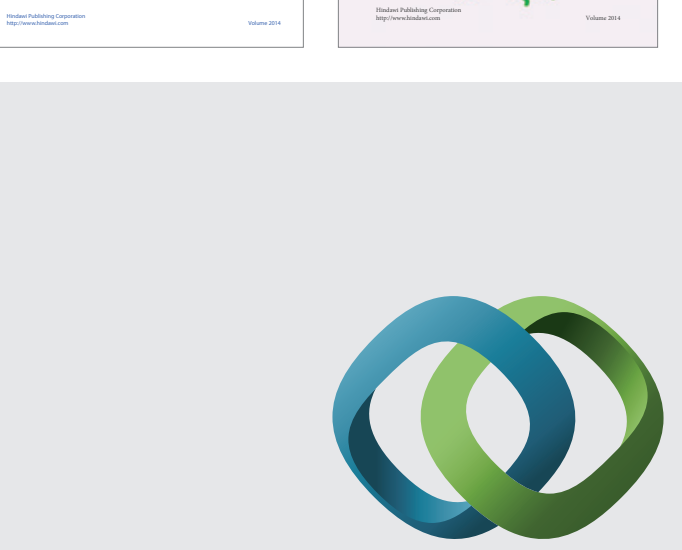

\section{Hindawi}

Submit your manuscripts at

http://www.hindawi.com
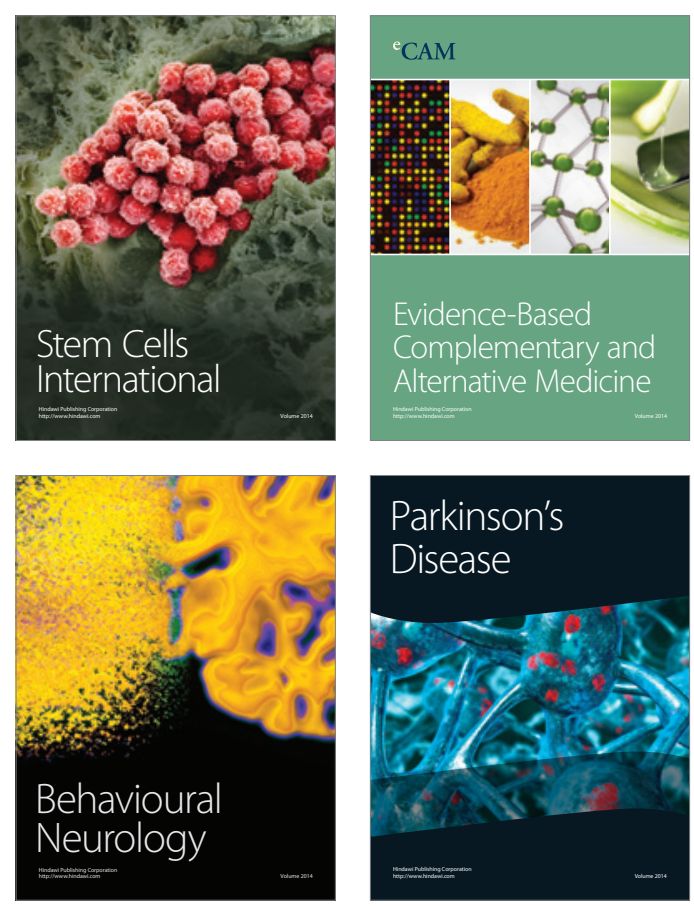

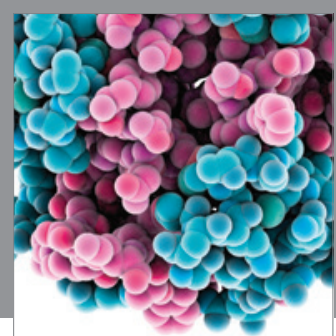

Journal of
Diabetes Research

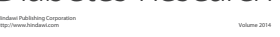

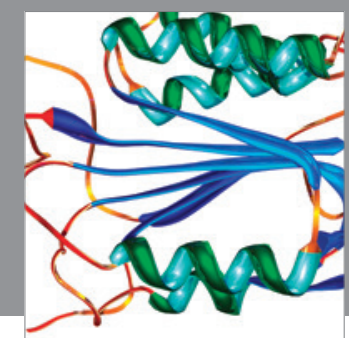

Disease Markers
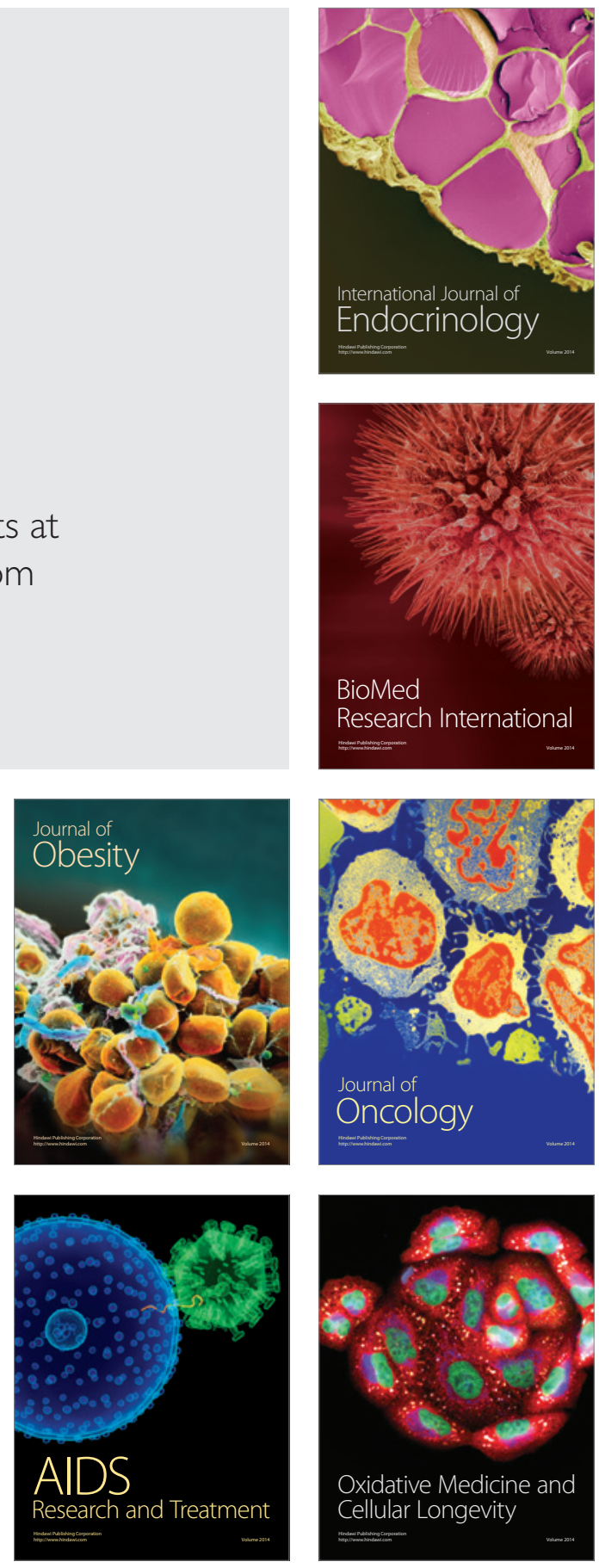